\title{
Using Beryllium and Polypropylene-Aluminium Absorbers to Measure the TBR-1 Tokamak Electron Temperature
}

\author{
K. A. de Oliveira, A. Vannucci, R. M. O. Galvão, \\ M. S. T. Araújo and I.C. Nascimento \\ Instituto de Física da Universidade de São Paulo \\ Caixa Postal 66318 - CEP: 05315-970, São Paulo, Brazil
}

Received June 2, 1998

\begin{abstract}
Beryllium foils of different thickness have been employed for measuring the electron temperature in tokamaks using the absorbing method based on ratio of the bremsstrahlung intensities, emited by the plasma. However, in the case of small tokamaks with low electron temperatures, such as TBR-1, the foils must be very thin, becoming very fragile, hard to handle and with insufficient absorbing power of line radiation from impurities. Also they are very expensive. In this work, we have investigated whether other absorber materials, obtained by depositing Aluminium on polypropylene films, for example, could give similar results. These new kind of filters have good mechanical properties and are light tight, as opposed to the thin beryllium foils which have shown, very often, the presence of pinholes. Also, the attenuation of the line emission from impurities, with the use of such filters, is more effective. Silver foils have also been manufactured and used, but with unsatisfactory results due to the TBR-1 low plasma temperature. However, silver foils should be considered a good candidate for measuring $T_{e}$ through the absorbing method in large tokamaks with higher plasma temperatures.
\end{abstract}

\section{Introduction}

The controlled thermonuclear fusion is a hope for the mankind of a cheap and inexhaustible source of energy. Towards this goal, intensive efforts have already been spent, during the last decades, in search for processes that could maintain the plasma magnetically confined while its temperature is strongly increased. Among the different magnetic confinement devices, the tokamak is the one that has achieved the best results up to now.

During the tokamak plasma discharges, several diagnostics are put in operation to measure the plasma parameters and monitor the plasma conditions. In particular, the soft X-ray detection system has become an important tool to investigate the central part of the plasma column, since the emission of low energy X-rays is closely related to the electron temperature and also the plasma density and impurities. In this paper, the use of pairs of soft X-ray detectors covered with foils of different thickness, for measuring the electron temperature of the plasma confined by the TBR- 1 tokamak, will be presented and discussed. Originally, this method of detecting radiation through materials of different thickness was first developed for biological purposes by Jahoda et al. in 1960 [1]. Latter on, this technique was applied to measure the electron temperature of plasmas magnetically confined and, till now, many works have already been carried out in many different tokamaks $[2,3,4]$.

The great advantage of this method is its cheapness, when compared to other more sophisticated systems as Electron Cyclotron Emission and Thomson Scattering. Also, it allows measurements of the electron temperature to be performed during the entire duration of the discharge and not only in specific instants of time, as for Thomson Scattering. Unfortunately, the two foils absorbing method is not as accurate as it would be desired, because it involves the calculation of integrals of 
exponential functions and also because it is very sensitive to line radiation emission from impurities present in the plasma. However, despite these drawbacks, it has already been shown that very good experimental results can be obtained through this technique [3] and it is certainly very much appropriate to be used in small tokamaks like the TBR-1, where only low plasma electron temperature is usually reached.

Traditionally, the thin foils used in front of the soft $\mathrm{X}$-ray detectors are made of beryllium which are rather expensive, extremely fragile ( for low electron temperature of the plasma ) and toxic. For this work, a new soft X-ray detection system for the TBR-1 tokamak was constructed in which different type of materials, other than beryllium, were alternatively used to measure the temporal profile of the plasma electron temperature. Initially, the filters were made of silver (obtained by evaporation) because it has the important characteristic of efficiently attenuating the VUV line radiation emitted by the plasma impurities (mainly carbon, oxygen and nitrogen). However, these silver filters were not found to be totally adequate for measuring the TBR1 central electron temperature, as it will be discussed later. Rather, the silver foils should be more suitably used to measure tokamak plasma electron temperatures above $300 \mathrm{eV}$.

Aluminium deposited in a thin polypropylene film was then found to be the best choice for an appropriate absorbing material to work with, providing satisfactory results when used to measure the TBR-1 central electron temperature. The Aluminium-polypropylene filter has shown good resistance against mechanical vibrations, it is not toxic and attenuates the line radiation more efficiently than beryllium; properties that determined its utilization in this work.

In result, the average TBR-1 central electron temperature, for plasma discharges that lasted from $8 \mathrm{~ms}$ to $10 \mathrm{~ms}$ and with plasma current around $12 \mathrm{kA}$, was measured to be around $130 \mathrm{eV}$. The corresponding average Zeff, using the Spitzer formula, was found to be 3.2 , approximately.

\section{X-Ray Emission}

The soft X-ray spectrum emitted by plasmas magnetically confined in tokamaks is basically composed of a continuum and some line radiation peaks over it. The continuum radiation is formed by two main components:

- Bremsstrahlung radiation, produced when energetic electrons interact with the impurity ions or with the hydrogen isotopes.

- Electron-ion recombination, related to the capture of a free electron by the ions in the plasma.

In the former process, considering that the speed of the electrons is much greater than of the ions, the interaction rate, that depends basically on the electron temperature $\left(T_{e}\right)$ and the production of bremsstrahlung radiation, on the soft $\mathrm{X}$-ray range, is given by [2]:

$$
\left(\frac{\Delta W}{\Delta E}\right)_{b r} \propto n_{e} \sum_{i} n_{i} Z_{i}^{2} T_{e}^{-\frac{1}{2}} e^{\frac{-E}{T_{e}}}
$$

where:

- $\Delta W$ is the radiated power, per $\mathrm{cm}^{3}$, corresponding to photons in the energy range $\Delta E$.

- $n_{e}$ and $n_{i}$ are the electron and ion densities, respectively.

- $Z_{i}$ is the atomic number of the ions.

- $E$ is the energy of the emitted photon.

The expression (1) shows that the emission spectrum has an exponential dependence with the electron temperature and it provides a way of calculating this temperature. Furthermore, since the plasma is hotter and denser at the central part of the plasma column, it can be concluded that most of the X-ray emission will came from this region of the plasma. The soft X-ray detecting system turns out to be, therefore, an excellent diagnostic to analyze this part of the plasma column.

The radiation due to the electron-ion recombination, in the energy range above the ionization potential of the atom that participates in the process, can be written as [2]: 


$$
\left(\frac{\Delta W}{\Delta E}\right)_{r e c}=\left(\frac{\Delta W}{\Delta E}\right)_{b r}(\gamma-1)
$$

since both emission processes have the same spectrum format. Hence, the parameter $\gamma$ reflects the enlargement of the bremsstrahlung spectrum due to the recombination radiation. The energy of the photon that is emitted during the recombination corresponds basically to the kinetic energy of the free electron plus the energy associated with the final atomic state of the bounded electron.

\section{Diagnostic System}

The soft X-ray detection system built to measure the electron temperature of the plasma confined by the TBR-1 tokamak is composed of four surface-barrier detectors placed, in pairs, on a flange about $40 \mathrm{~cm}$ away from the plasma (Fig. 1). The electronic signals, after the pre-amplification, were digited by CAMAC modules at the sampling rate of $400 \mathrm{kHz}$.

TRANSVERSAL SECTION OF TBR-1

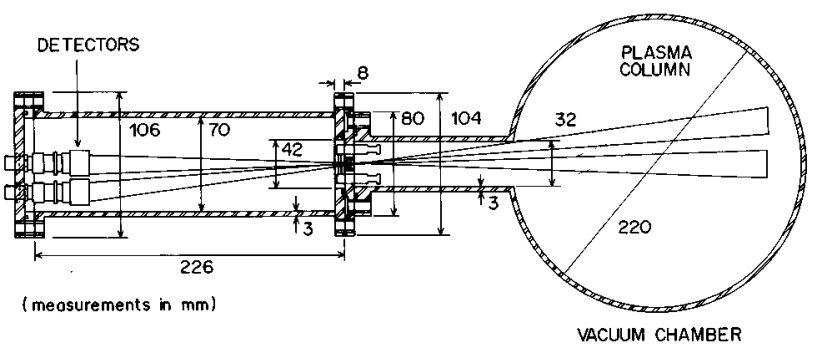

Figure 1. Soft X-ray detecting system with two pairs of surface-barrier detectors. One pair of detectors views de central plasma column while the other pairs views a region of about $2.0 \mathrm{~cm}$ above of the central region.

\section{III.1 Detectors}

The surface-barrier detectors (SBD) used, manufactured by ORTEC, have ruggedized frontal electrodes made of Aluminium. This type of detector has high detection efficiency in the soft $\mathrm{X}$-ray region, good temporal resolution and is easy to manipulate. This semiconductor device is built by evaporating Aluminium on the front surface of a thin silicon slab, creating the $\mathrm{p}-\mathrm{n}$ junction. Each photon that reaches the sensitive layer inside the silicon creates a pair hole-electron for each $3.6 \mathrm{eV}$ of energy that is transferred to the silicon. The number of photons absorbed in the depletion region, with thickness that is a function of the difference of the potential applied and also of the intrinsic resistivity of the material, depends on the transmissibility of the metal deposited in the silicon front surface, on the silicon dead layer and, finally, on the transmissibility of the filters that are placed externally in front of the detector [3]. All these considerations must be taken into account to determine how the original spectral distribution of the radiation, that come from the plasma, is modified. The specifications of the detectors used are:

- Aluminium evaporated on the front surface: 0.15 $\mu m$

- Thickness of the silicon dead layer: $0.01 \mu \mathrm{m}$

- Thickness of the depletion region: $100 \mu \mathrm{m}$

- Polarization voltage (negative): $12 \mathrm{~V}$

Each surface-barrier detectors works essentially as a current source in parallel with a capacitance of about $50 \mathrm{pF}$ (at $12 \mathrm{~V}$ bias). The detectors are connected, by a short coaxial cable, to current-to-voltage preamplifiers which ultimately determines the frequency response and, also, the noise performance of the system. The electronic circuits of the preamplifiers are shown in Fig. 2 and 3. The upper circuit frequency response is determined by the feedback capacitors $C_{1}$ and $C_{2}$. They give a theoretical $3 \mathrm{~dB}$ bandwith of about 100 $\mathrm{kHz}$, which is suitable to our application. The strongest noise component is due to the thermal noise in the feedback resistor $R_{1}$ and also the noise that comes from the first stage of the operational amplifier. In general, the noise level for LF-357 operational amplifier is about $1 \mathrm{pA} / H z^{\frac{1}{2}}$ in the frequencies of interest [3].

The relative calibration of each pair of detectors was done by placing absorbing foils of the same thickness in front of both detectors and comparing the detector output signal amplitudes. 


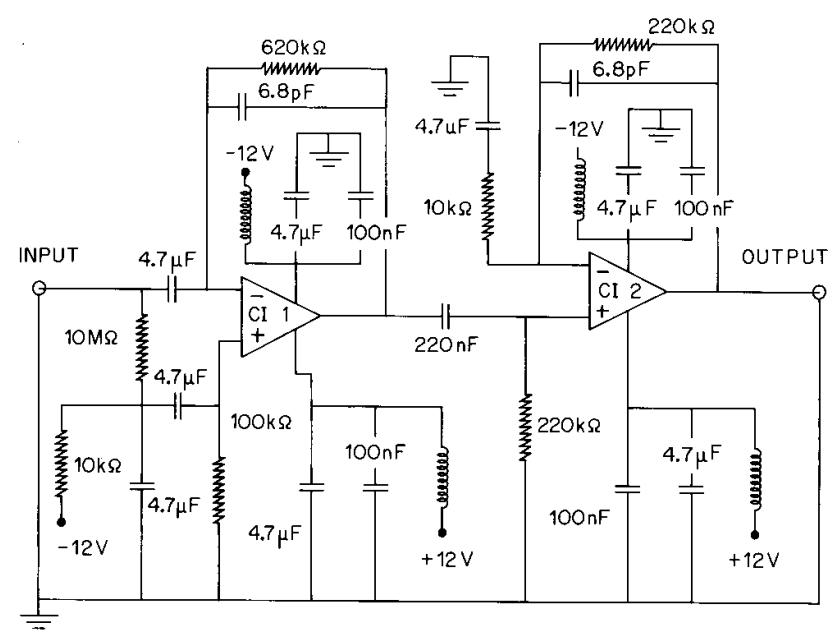

Figure 2. Electronic circuit of the preamplifier used to measure the soft $X$ - rays signals using beryllium filters. The inductors were added to reduce de electronic noise.

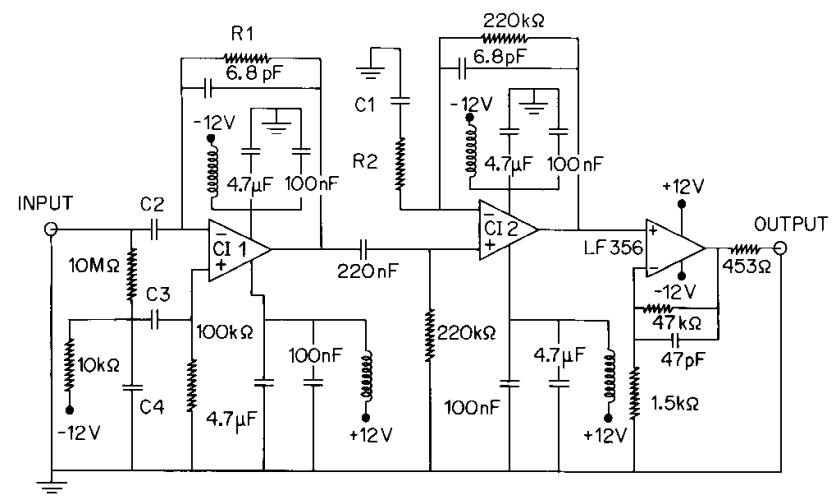

Figure 3. Electronic circuit of the preamplifier used to measure the soft X - rays signals using polypropylene - Aluminium filters. In this particular case, it was necessary to add one more amplification stage to detect the low intensity signals.

\section{III.2 Electronic Noise}

In order to take into account the possibility of picking up some external electromagnetic noises, that could affect our measurements, the hydrogen filling gas flux of the tokamak was turned off after a sequence of normal discharges, and the capacitor bank was then fired. This procedure allowed us to obtain a tokamak discharge with no plasma and it was performed, basically, everytime the initial discharge conditions were modified. Afterwards, both signals obtained from pulses with and without plasma are subtracted from each other. This subtraction is performed by running a computer code which also calculates, sequentially, the ratio of the radiation intensities and which, finally, gives the temporal profile of electron temperature.

\section{The Absorbing Foils Method}

The method of measuring the plasma electron temperature by using absorbing foils consists in taking the ratio, $\mathbf{R}$, of soft $\mathrm{X}$-rays intensities measured by a pair of surface-barrier detectors, with filters of two different thicknesses placed in the optical path of the detectors, each one viewing the same thermal region of the plasma column through equal solid angles (Fig. 4).

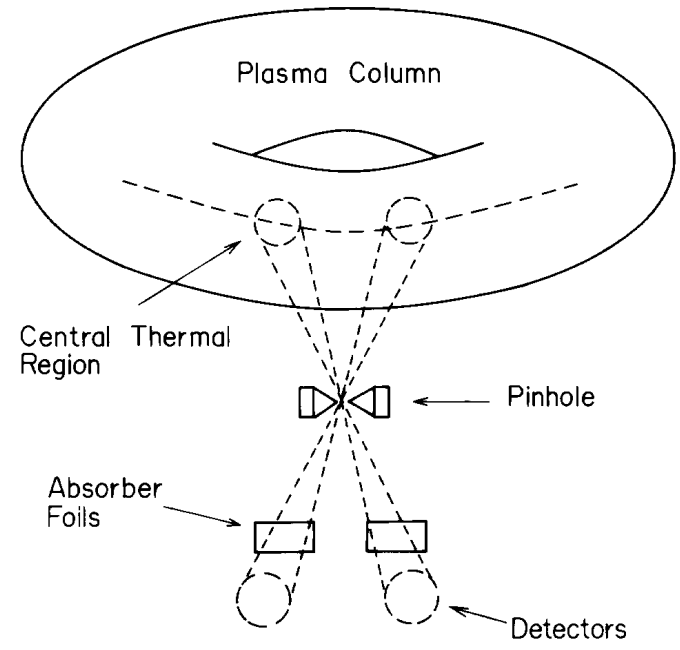

Figure 4. Illustrative sketch of the experimental arrangement with a pair of surface-barrier-detectors viewing the same thermal region of the plasma column.

The use of this method is based on two basic assumptions: first, the intensity of the bremsstrahlung radiation per unit of wavelength interval, emitted in the $\mathrm{X}$-ray region by the hot plasma, varies exponentially with the electron temperature and second, the $\mathrm{X}$-ray mass absorption coefficients are energy dependent, and the coefficients, for several different materials commonly used as filters, are experimentally known and can be found in the literature [5].

Taking also into account the absorbing materials which are part of the detectors that were used in our experiment, as the $\mathrm{Al}$ front electrode (thickness of 0.15 $\mu \mathrm{m}$ ) and the Si dead-layer (thickness of $0.01 \mu \mathrm{m}$ ), besides the foil placed in front of each detector, we can write [4]: 


$$
R=\frac{\int \exp \left[\frac{-h e}{\lambda T_{e}}-\mu_{F_{1}} x_{F_{1}}-\mu_{A l} x_{A l}-\mu_{S i} x_{S i}\right] E_{f}(\lambda) \lambda^{-2} d \lambda}{\int \exp \left[\frac{-h e}{\lambda T_{e}}-\mu_{F_{2}} x_{F_{2}}-\mu_{A l} x_{A l}-\mu_{S i} x_{S i}\right] E_{f}(\lambda) \lambda^{-2} d \lambda}
$$

where:

$\mu \equiv$ mass absorbing coefficient of the used materials.

$\mathrm{x} \equiv$ filters thickness.

$\lambda \equiv$ wavelength of incident radiation.

$E_{f}(\lambda)=1-\exp \left(-0.01 \mu_{S i}\right) \equiv$ efficiency of the detector.

hc $\equiv 1.2398(\mathrm{eV} \mu \mathrm{m})$.

The integrals in equation (3) can be easily calculated, if a smooth function that gives the mass absorbing coefficient for each of the incident radiation wavelengh can be found. It is already known that the best fit for the mass absorption coefficient of the materials obtained from reference [5], in the wave length interval up to $0.01 \mu \mathrm{m}$, is of the type [6]:

$$
\mu(\lambda)=A \cdot \lambda^{(B+C \ln \lambda)},
$$

where:

$\lambda \equiv$ wavelength of incident radiation.

$\mathrm{A}, \mathrm{B}$ and $\mathrm{C} \equiv$ adjustable parameters.

The numerical calculation of the expression (3), writting the mass absorption coeficients as equation (4), was done for 30 arbitrary $T_{e}$ values from 50 to $300 \mathrm{eV}$, considering the filter thicknesses shown in table 1 :

\begin{tabular}{|c|c|c|c|c|c|c|}
\hline \multirow{2}{*}{ Foils } & \multicolumn{6}{|c|}{ Thicknesses in $(\mu m)$} \\
\cline { 2 - 7 } & $x_{F_{1}}$ & $x_{A l_{1}}$ & $x_{S i}$ & $x_{F_{2}}$ & $x_{A l_{2}}$ & $x_{S i}$ \\
\hline Be & 0.20 & 0.15 & 0.01 & 0.50 & 0.15 & 0.01 \\
Ag & 0.33 & 0.15 & 0.01 & 0.52 & 0.15 & 0.01 \\
Poly. + Al & 0.84 & 0.16 & 0.01 & 1.05 & 0.30 & 0.01 \\
\hline
\end{tabular}

Thicknesses of used filters for numerical calculations employed.

The adjusted curves for $\mathbf{R}$ (third degree polynomials) were obtained for different type of filters used in this work, and are showed in Fig. 5. Note that in this figure the ratio $\mathbf{R}$ varies monotonically with the electron temperature values, for beryllium and polypropylene-Aluminium foils, providing a good discrimination in the range $100-300 \mathrm{eV}$, in relation to the
X-ray flux experimentally measured. However, we don't find the same performance with silver foils.

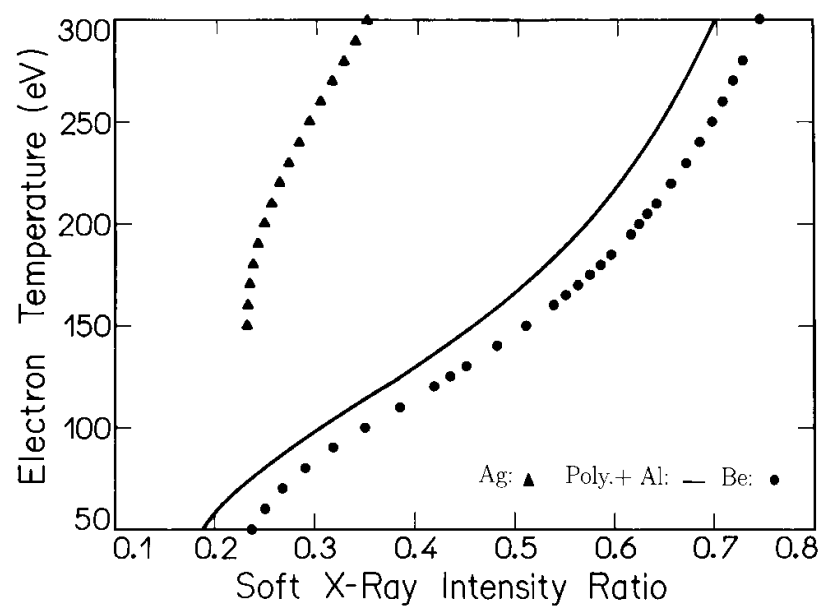

Figure 5. Electron temperature versus soft $X$ - ray intensity ratio, for beryllium foils (circle symbols), polypropylene Aluminium foils (continuous line) and silver foils (triangle symbols). The calculated points were adjusted by a polynomial function of the third degree.

\section{Manufacturing the filters}

The absorbing foils that replaced the beryllium filters, for this experiment, were constructed at the Physics Institute of the University of São Paulo and at the Institute for Advanced Studies (IEAv). Different techniques were used to obtain each kind of foil. The thickness of the filters were previously estimated, in each case, as to give the best ( $\mathrm{R} \times \mathrm{Te}$ ) curve slope for the TBR-1 electron temperature range.

\section{V.1 Silver Filters}

Silver filters with thickness of about $0.30 \mu \mathrm{m}$ and $0.50 \mu \mathrm{m}$ were obtained by standart deposition technique and mounted in a circular support, as a free standing foil. The exact thickness of the foils were measured by placing them in a vacuum chamber and measuring the energy lost by incident Alfa particles [7, 8].

\section{V.2 Aluminium Coated Polypropylene Films}

Thin polypropylene films were obtained by manual extrusion. Thicknesses of about $0.80 \mu \mathrm{m}$ could be easily obtained, as recognized by the blue color of the 
film, caused by the reflecting light interference. Precise values of the thicknesses and also good homogeneity of the films are important factors for the accuracy of the method. Quantitatively, the thickness of the foils were obtained by analyzing the interference fringes that result from the superposition of the light reflected in both surface boundaries of the polypropylene film. Hence, the index of refraction and the thickness of the film could be obtained by using a spectrophotometer which measures the light transmitance in relation to the wavelength, or wave number, of the incident photons. The equipament used for this purpose was a Lambda $9 \mathrm{UV}$ - Visible - Near Infrared spectrometer where two radiation sources, a deuterium lamp and a halogen lamp, covered the working wavelength rang of the espectrometer [9]. The analysis of the spectrum obtained indicated how uniform were the plastic supports obtained by extrusion. Fig. 6-a, for example, shows the spectrum obtained for a very homogeneous film while, in Fig. 6$\mathrm{b}$, the spectrum obtained for a non-homogeneus film is observed. Note that, in the latter case, the maximum and minimum peaks are not well defined.

This method of analysis allowed the measurements of the thickness $\mathbf{h}$ and the refractive index $\mathbf{n}$ of the polypropylene films to be obtained using the following relation, for normal incidence of the light [9]:

$$
h=\frac{m}{4 n \sigma}
$$

where:

$$
\begin{aligned}
& \mathrm{h} \equiv \text { thickness of the plastic film. } \\
& \mathrm{m} \equiv \text { interference order. } \\
& \sigma \equiv \text { wave number of the monocromatic inci- }
\end{aligned}
$$
dent light.

$$
n \equiv \text { index of refraction of the plastic film. }
$$

The index of refraction of the material can be obtained directly from the spectrum by using the expression:

$$
n=\sqrt{\frac{1+\sqrt{1-T}}{1-\sqrt{1-T}}},
$$

where $T$ is the intensity radiation transmited through the plastic film.

We have calculated the refractive index for 15 polypropylene films and obtained $\left\langle n_{\text {poly }}\right\rangle=1.47$ \pm 0.02 . This value is in very good agreement with the result found in the literature: 1,49 [10].
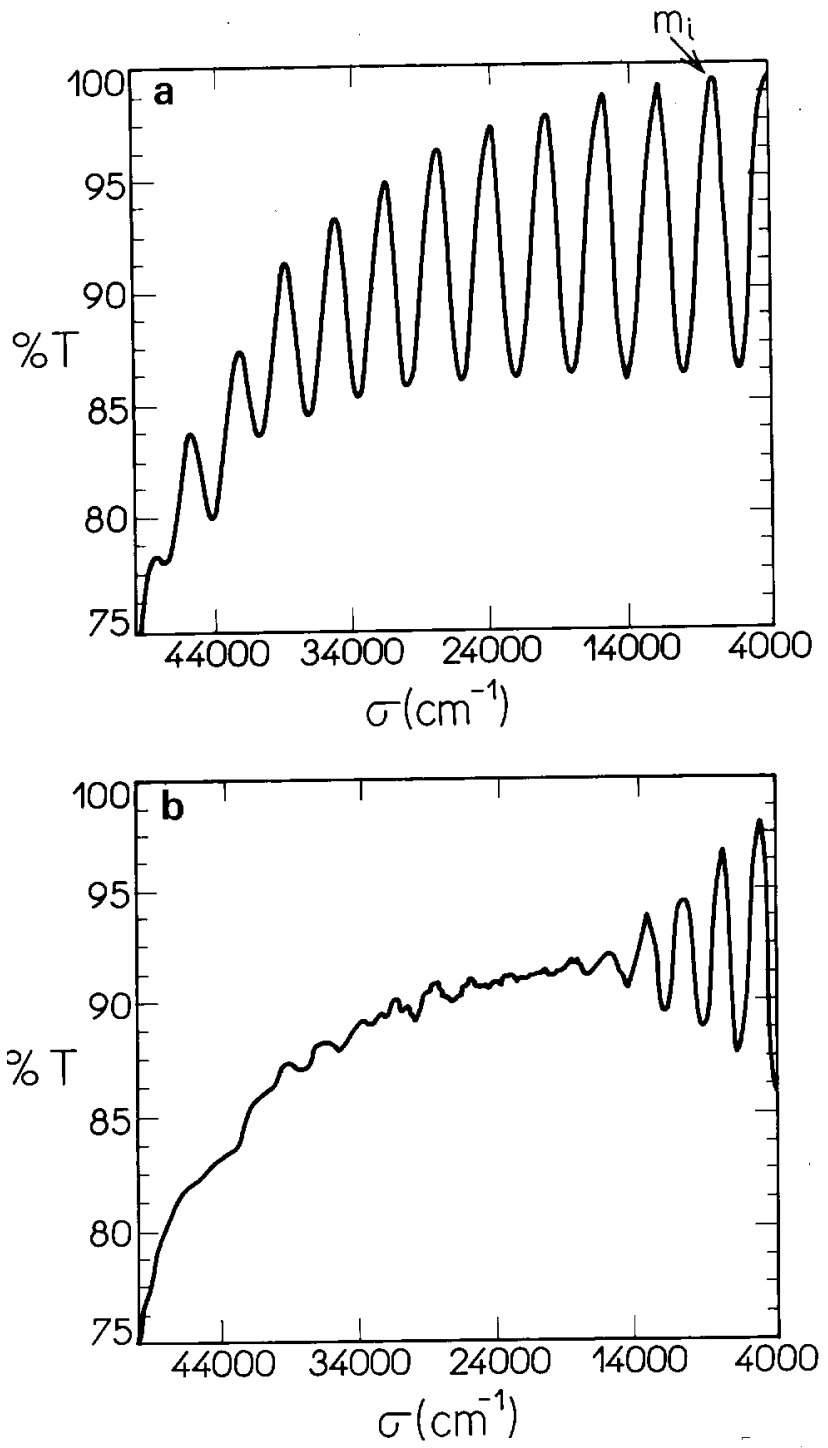

Figure 6. Spectrum obtained with a homogeneous polypropylene film (a), when only one color is observed to be reflected from the plastic film surface, and spectrum obtained with a non-homogeneous polypropylene film (b), when several colors are observed to be reflected from the plastic surface.

Polypropylene films were covered with Aluminium by standart deposition technique, with dinamic thickness deposition control (quartz cristal oscillation) [7].

\section{V.3 The transmissivity of the Filters}

The transmissivity curves for the beryllium, silver and polypropylene coated with Aluminium films, in function of the energy, are illustrated on Figures 7-a, 
7-b, and 7-c, respectively. As it can be observed, beryllium films (Fig. 7-a) are not suitable to be used for measuring low temperature plasmas, due to the large existing transmissivity window between $30 \mathrm{eV}$ and $70 \mathrm{eV}$, where most of the line radiation is more likely to exist. In this respect, silver films (Fig. 7-b) seem to be more appropriate to attenuate the V.U.V. emission. However, they do not apropriately discriminate the plasma electrons temperature in relation to the radiations intensity values obtained, i.e., small changes in the soft $\mathrm{X}$-ray intensity ratio yields very large changes in $T_{e}$ values (Fig. 5). For polypropylene plus Aluminium films (Fig. 7-c) the transmissivity in this range of energy is decreased by an order of magnitude, indicating that these filter are appropriate to be used in small tokamaks such as the TBR-1.

\section{Experimental Results}

Figures 8-9 and 10-11 show the basic experimental signals (plasma current $\left(I_{p}\right)$, loop voltage $\left(V_{l}\right)$, central soft X-ray $(r x)$ and hard X-ray $(R X)$ obtained for two different plasmas pulses. The discharge shown in Figures 8-9 corresponds to a maximum plasma current of about $10 \mathrm{kA}$, that lasted for about $7 \mathrm{~ms}$. In this discharge the central electron temperature was measured during the flat top portion of the plasma current, using beryllium and silver foils. The results obtained are presented in Fig. 12 and Fig. 13. As one can see, with berillium foils the central electron temperature pratically remains the same $\left(T_{e} \sim 140 \mathrm{eV}\right)$ along the $2 \mathrm{~ms}$ of the current plateau. After that, $T_{e}$ could not be calculated because strong bursts of hard X-ray emission were observed, related to the formation of runaway electrons (Figure 8-c), which turned this method of analysis impracticable. The central electron temperature measured using silver foils are presented in Figure 13. As we can see, the temperature profile is very different from the one that measured by using beryllium foils, althought both X-ray signals have been simultaneously measured. Analysing the Figure 5 one observes that the calibration curve, for silver foils presents a very high slope that explain the many oscillations observed in the electron temperature profile. This might explain, also, the large electron temperature value $(\sim 300 \mathrm{eV})$ which is unrealistic for small size tokamaks as the TBR1. Figure 14 shows the central electron temperature obtained with polypropylene-Aluminium foils. As we can see, the obtained $T_{e}$ profile presents many oscillations, reflecting the strong fluctuations observed in the raw soft X-ray signals. However, the average value of about $130 \mathrm{eV}$ is more realistic and it is in a fair agreement with the results obtained with the beryllium films.

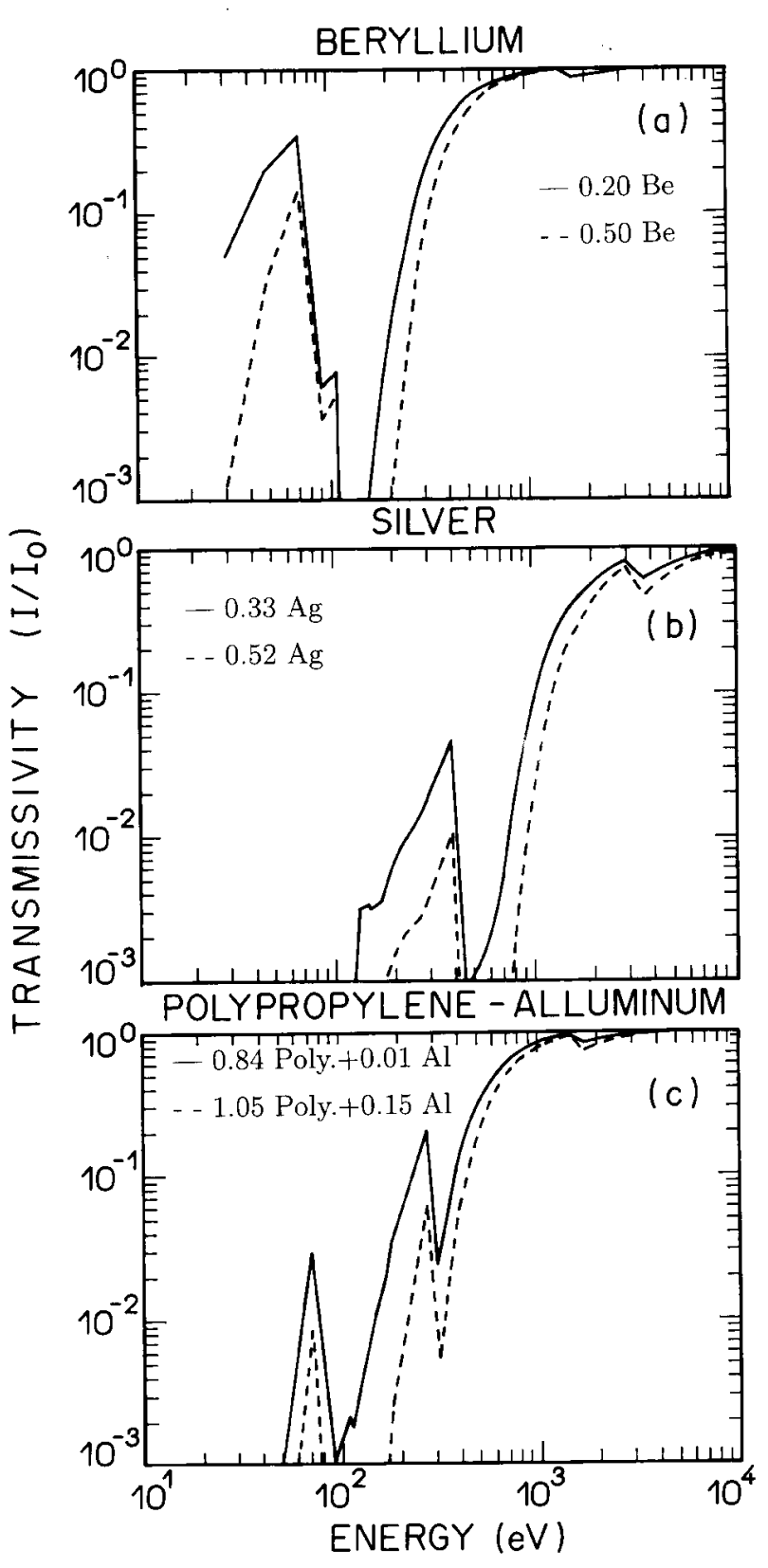

Figure 7. (a) Transmissivity of beryllium foils: $0.20 \mu \mathrm{m}$ (continuous line) and $0.50 \mu \mathrm{m}$ thickness (dashed line);(b) silver foils: $0.33 \mu \mathrm{m}$ (continuous line) and $0.52 \mu \mathrm{m}$ thickness (dashed line); (c) polypropylene-Aluminium foils: 0.84 $\mu \mathrm{m}$ polypropylene plus $0.01 \mu \mathrm{m}$ Al thickness (continuous line) and $1.05 \mu \mathrm{m}$ polypropylene plus $0.15 \mu \mathrm{m}$ Al (dashed line). 
Shot G130

Shot G130
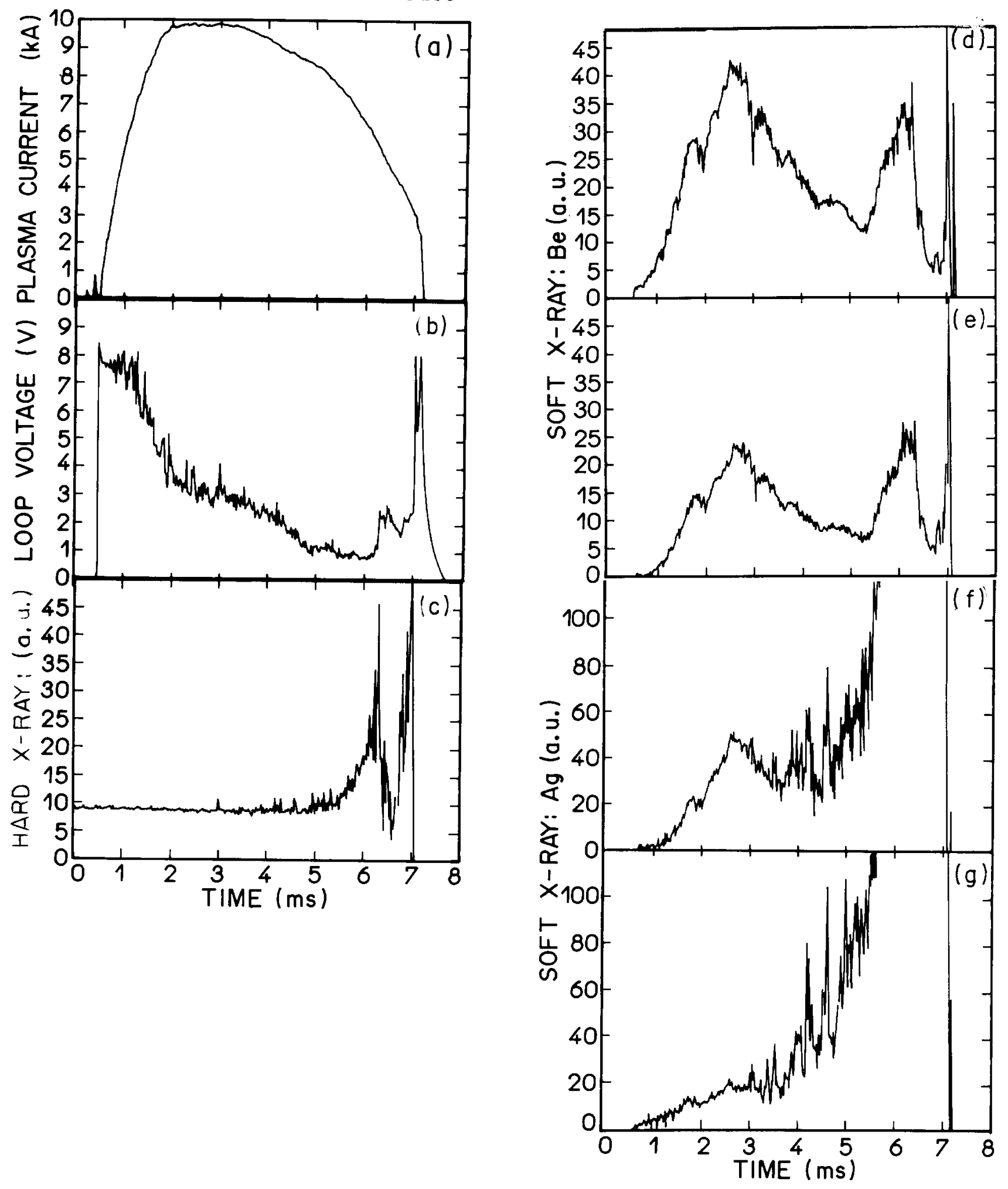

Figure 9. Soft X - ray signals measured by a pair of detectors which view a central region of the plasma column with beryllium foils (figs: (d) and (e)) and with silver foils (figs: (f) and (g)).

Figure 8. Plasma current (fig.a), loop voltage (fig.b) and hard X-ray (fig.c) from plasma pulse G130. 
Shot G133
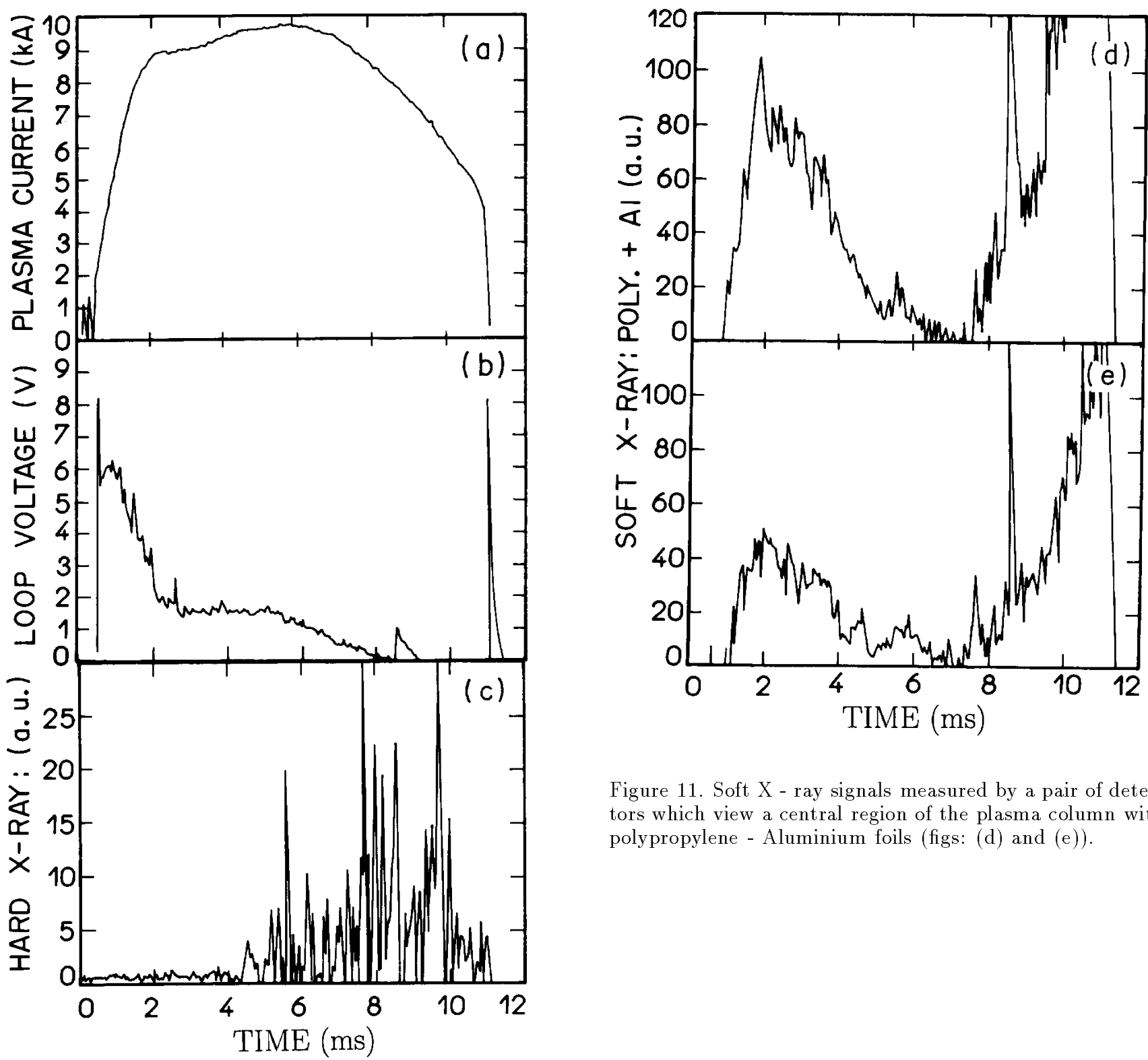

Figure 11. Soft X - ray signals measured by a pair of detectors which view a central region of the plasma column with polypropylene - Aluminium foils (figs: (d) and (e)).

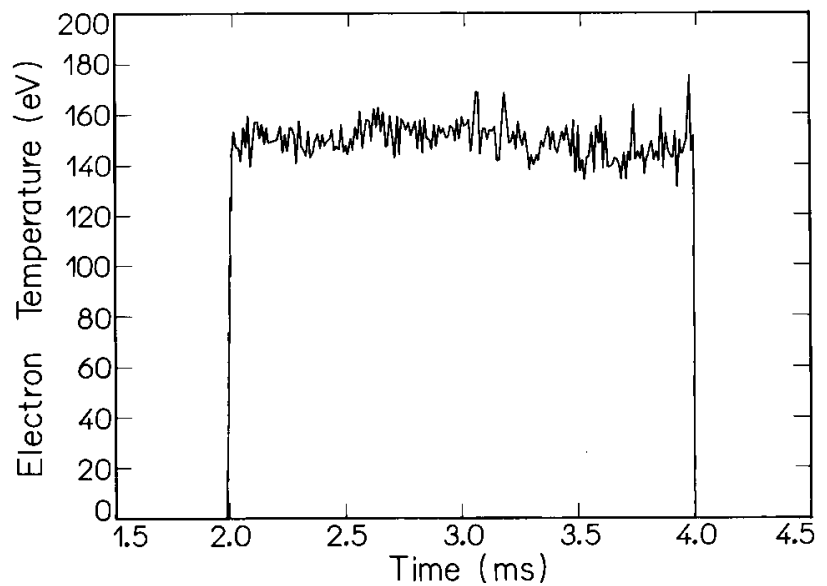

Figure 10. Plasma current (fig.a), loop voltage (fig.b) and hard X-ray (fig.c) from plasma pulse G133.

Figure 12. Electronic temperature of the central plasma column obtained by the absorber method using beryllium foils. 


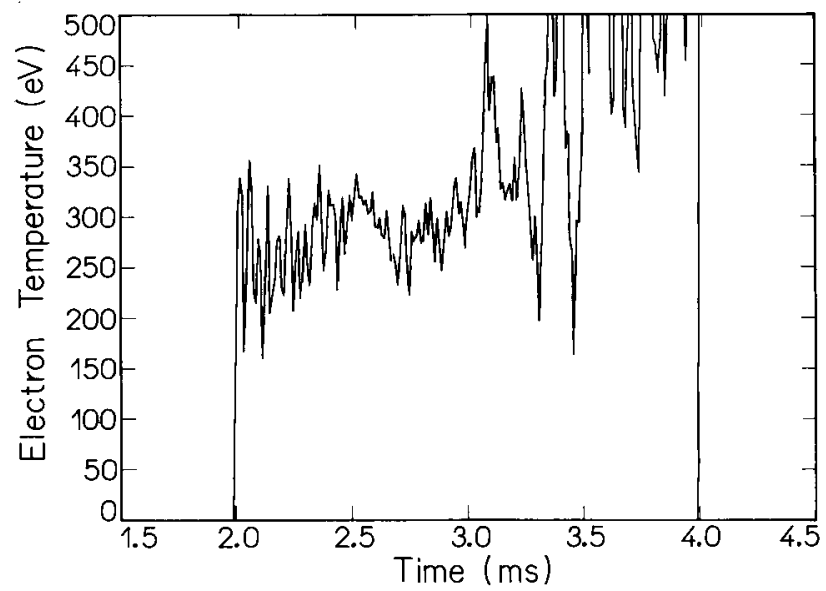

Figure 13. Electronic temperature of the central plasma column obtained by the absorber method using silver foils.

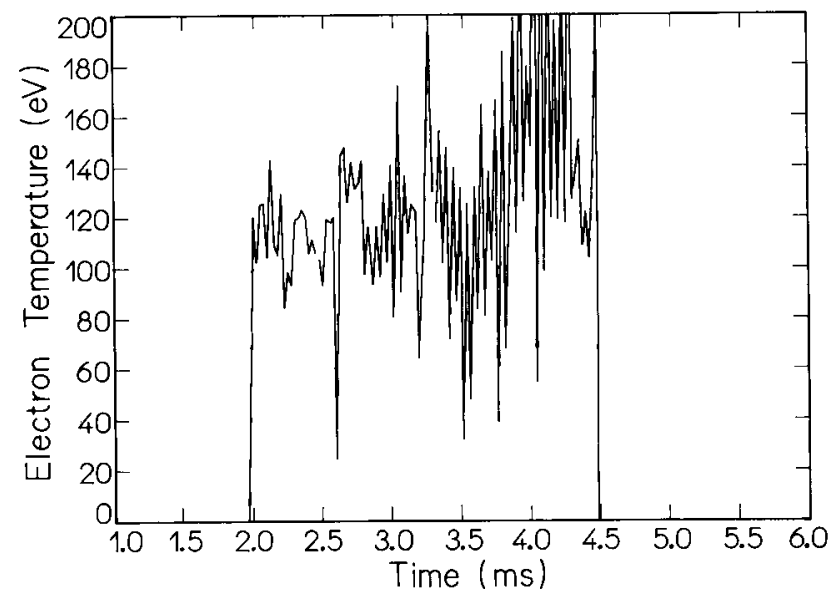

Figure 14. Electronic temperature of the central plasma column obtained by the absorber method using polypropylene - Aluminium foils.

\section{Conclusion}

For this work, very suitable soft X-ray filters were obtained by evaporating Aluminium over a polypropilene film, which replaced, with advantages, the imported beryllium filters that have been utilized for measuring the TBR-1 electron temperature. Contrary to beryllium, the Aluminium-polypropilene filter are very strong against mechanical vibrations, easy to manufacture, non-toxic and considerably much cheaper.

Experimentally, the TBR-1 electron temperature measured by the two foils method, using the new filters, ranged from $100-130 \mathrm{eV}$ for tokamak operation conditions: plasma current: about $10 \mathrm{kA}$, plasma discharge: approximately $8 \mathrm{~ms}$, gas filling pressure: $6,0 x$ $10^{-5}$ mbar. The TBR-1 central electron temperature measured with the Aluminium-polypropilene filters resulted to be somewhat smaller than the temperature obtained by using the beryllium foils. If one considers that very thin beryllium filters usually have very tiny holes which may affect all the calculations and, also, that beryllium is not so efficient to absorb the line radiation emitted by the impurities, then it is reasonable to accept that the values obtained with the use of Aluminium-polypropilene filters are more precise. The corresponding average Zeff for the TBR-1 plasma, using the Spitzer formula, was found to be 3.2 , approximately. The silver films, although being as simple to obtain as the Aluminium-polypropilene filters, were not considered to be a good choice for measuring the electron temperature of low temperature plasmas, because of the (Te $\mathrm{x} \mathrm{R}$ ) slope, which is too steep for temperatures bellow $300 \mathrm{eV}$. This kind of filter, however, may give good results for plasmas with electron temperature above $300 \mathrm{eV}$.

\section{Acknowledgements}

The authors are grateful to the staff of the Laser Laboratory of the UNICAMP, where measurements of the polypropylene thickness were done, and to IEAv, for the helpful advisements about Aluminium deposition on the polypropylene filters.

\section{References}

[1] F.C.Jahoda, E.M.Little, W.E.Quinn, G.A.Sawyer and T.F.Stratton; Phy. Rev. 119, 843 (1960).

[2] S. von Goeler, Diagnostic for Fusion Experiments, Pergamon Press, N.Y., 79-109 (1979).

[3] M.Malacarne, I.H.Hutchinson, Soft X-Ray Imaging Diagnostic on the HBTX IA Reversed Field Pinch, Culhan Laboratory Report, CLM-R 227, England (1982).

[4] A.Vannucci et al., Nucl. Instr. and Meth. Phys. Research, A280, 593 (1989).

[5] B.Henke at al, Atomic Data and Nuclear Data Table, $1,27(1982)$.

[6] L.Nenu, E.Cojocaru, I.N.Mihailescu, Filter Method for Electron Temperature Determination from X-Ray Continuum Radiation Emitted by Hot Plasmas, Report LOP-10-80, Bucharest (1980).

[7] L. Holland, Vacuum Deposition of Thin Film, London, Chapman and Hall Ltd (1965). 
[8] J.F.Dias and M.N.Martins, Manual para Medida de Espessura de Alvos por Irradiação de Partículas Finas, Laboratório do Acelerador Linear Report, IFUSP/P839, Brasil (1990).

[9] M.Born and E.Wolf, Principles of Optics, $5^{\text {th }}$ edition,
Pergamon Press, Oxford, (1975).

[10] CRC Handbook of Chemistry and Physics, $58^{\text {th }}$ edition, CRC Press,INC (1969) C794.

[11] TFR Team, Nucl. Fusion, 213,17,2 (1977). 